\title{
DESARROLLO Y EVALUACIÓN DE UN MATERIAL DIDÁCTICO MULTIMEDIA PARA FACILITAR EL APRENDIZAJE DE MATEMÁTICAS
}

\section{DEVELOPMENT AND EVALUATION OF A MULTIMEDIA TEACHING MATERIALS TO FACILITATE MATHEMATICS LEARNING}

Santiago Valbuena Rodríguez ${ }^{1,4} \bullet$ Carlos Ortiz Gutiérrez² $\bullet$ Oscar Agudelo Varela ${ }^{3}$

\section{RESUMEN}

En el presente estudio se desarrolló un material didáctico multimedia que integra los componentes de tecnología y pedagogía con el fin de apoyar el proceso de enseñanza y aprendizaje de matemáticas para el programa de Ingeniería Electrónica de la Universidad de los Llanos. En el diseño se aplicó la metodología de Ingeniería del Software Educativo, y se utilizó la Suite Adobe. La evaluación cualitativa del material se realizó por usuarios con prueba piloto y una prueba de campo, evaluación de un experto en contenidos de matemáticas. La evaluación experimental se basó en inferencia estadística, mediante la prueba de comparación t de Student, a través de un examen de conocimientos entre un grupo de estudiantes de ingeniería electrónica de un curso de matemáticas I de enseñanza tradicional y otro grupo del mismo curso que trabajó con el multimedia de matemáticas terminado. El material producido promueve la interactividad docente-contenidosestudiante y el autoaprendizaje de Matemáticas I, de acuerdo con lo expresado por parte de los usuarios y el resultado favorable de la evaluación realizada por el experto. El resultado de la prueba t de Student, mostró que el promedio más alto de calificación lo obtuvieron los estudiantes del curso que trabajó con el multimedia de matemáticas desarrollado.

Palabras clave: aprendizaje, pedagogía, interactividad, multimedia

Químico, M.Sc, Coordinador Grupo: "Horizonte Mediático", svalbuena@unillanos.edu.co

2 Matemático. M.Sc. Grupo de Investigación Horizonte Mediático, Facultad de Ciencias Básicas e Ingeniería, Universidad de los Llanos, Villavicencio, Colombia.

3 Ingeniero de Sistemas, Esp. M.Sc. Grupo de Investigación Horizonte Mediático, Facultad de Ciencias Básicas e Ingeniería, Universidad de los Llanos, Villavicencio, Colombia.

4 Autor para correspondencia: svalbuena@unillanos.edu.co, hmediatico@unillanos.edu.co 


\section{ABSTRACT}

In this research was developed a multimedia learning material that the components of technology and pedagogy with the purpose of support the teaching and learning process of mathematics I, for the Electrical Engineering program at the Universidad de los Llanos. In the design, the methodology of Educational Software Engineering was applied and also was used Adobe Suite. The qualitative evaluation was made for user by the middle of a pilot test and a field test, evaluation of an expert in mathematics content. The experimental evaluation was based on statistical inference, by comparison test Student t, through a knowledge test among a group of electrical engineering students of mathematics course I of traditional teaching and another of the same course that worked with multimedia mathematics finished. The material produced promotes teacherstudent-content interactivity and self-learning of Mathematics I, according to the statement made by the users and the favorable outcome of the assessment by the expert. The result of the t test showed that the highest average grade obtained at the course the students worked with the multimedia math developed.

Key words: learning, teaching, interactivity, multimedia

\section{INTRODUCCIÓN}

Las dificultades de aprendizaje se definen como un problema o trastorno en el habla, lenguaje, escritura, aritmética u otras áreas escolares que resulta de una discapacidad provocada por una disfunción cerebral o alteración emocional o conductual, que no es resultado de retraso mental, por supresión sensorial o factores culturales e instruccionales, (Kirk y Bateman, 1962). En 1990 la National Joint Committee on Learning Disabilities NJCLD, se refiere a las dificultades de aprendizaje DA, como un grupo heterogéneo de trastornos que se manifiestan por dificultades significativas en el habla, la lectura, la escucha, la escritura, razonamiento o habilidades matemáticas.

El Manual Estadístico de trastornos mentales DSM-IV-TR y la Clasificación Internacional de Enfermedades CIE-10, definen las dificultades de aprendizaje como trastornos del aprendizaje, que en el caso de las matemáticas es un trastorno del cálculo.
La discalculia es el término que se refiere a las dificultades de aprendizaje de las matemáticas DAM y es un trastorno de habilidad para calcular. Esta, se ha estudiado especialmente en niños desde la psicología y la neurociencia, caracterizándose por la dificultad para memorizar y entender datos numéricos o aritméticos, así como para realizar cálculos y generar ideas en la solución de problemas (Jordan y Hanich, 2000; Mazzoco y Myers, 2003; Landerl et al., 2004).

En el diagnóstico del trastorno del cálculo la DSM-IV-TR establece tres criterios específicos: uno, cuando el alumno en los exámenes de matemáticas presenta resultados con dos desviaciones por debajo de lo esperado; dos, en el caso de que la dificultad de aprendizaje afecta el rendimiento académico; y tres, cuando este problema no se puede explicar por una deficiencia sensorial.

Cuando el problema de aprendizaje del cálculo produce bajo rendimiento académico, se genera 
deserción estudiantil, que según el Sistema para la Prevención de la Deserción en las Instituciones de Educación Superior, SPADIES, del Ministerio de Educación Nacional de Colombia, en el año 2008 fue del 44.9\%; en el año 2012 alcanzó el 45.3\% y en el 2013 un 44.9\%, (MEN, 2013); se señala también que por áreas de conocimiento, las matemáticas y las ciencias naturales, son las disciplinas que presentan la mayor tasa de deserción con un 51\% por cohorte en el nivel universitario, (MEN, 2013).

Esta deserción estudiantil tiene un efecto negativo en las instituciones universitarias, entre estas se encuentra la Universidad de los Llanos porque no permite el cumplimiento de la misión institucional de ayudar a formar ciudadanos Colombianos, generando enormes pérdidas económicas para las familias de los jóvenes implicados y para el estado, por los recursos invertidos por cada uno de ellos, además tiene un efecto social negativo por la frustración de muchos jóvenes que no logran terminar su proyecto de vida.

Un aporte a la solución de la problemática de aprendizaje de las matemáticas en los últimos 30 años se viene realizando con la integración de las Tecnologías de la Información y la Comunicación, TIC, la cual permite enfoques dinámicos en los conceptos principales de álgebra y del cálculo, así como la vinculación de múltiples representaciones, favoreciendo la interactividad, generando un potencial enorme hacia la construcción de significados en contraste con las prácticas tradicionales de lápiz y papel (Ferrara et al., 2006).

Los sistemas algebraicos computarizados, SAC, sirven como instrumento pedagógico de ayuda en el proceso de enseñanza y aprendizaje de las matemáticas, (Kutzler, 2003). Los SAC, facilitan las representaciones simbólicas, numéricas y gráficas de funciones y el desarrollo de cálculos numéricos, estadísticos, vectoriales y de matrices (Kendal. 2001). Existen varias versiones de los SAC, como el Mathematica, el
Maple y el Derive. Según Kumar (2007), el Mathematica facilita comprender los conceptos y el desarrollo de habilidades en la resolución de problemas de cálculo diferencial. El Derive ayuda a entender el concepto de integral definida (Camacho y Depool, 2003; Depool, 2004; Villareal, 2004).

Otro recurso de apoyo son los Applets elaborados con el programa de geometría Geogebra que ofrecen la posibilidad de hacer diferentes representaciones geométricas dinámicas e interactivas, que permiten la construcción de conceptos matemáticos, al promover la habilidad del pensamiento analítico y visual, necesario en la comprensión del cálculo diferencial e integral, (Aranda y callejo, 2010).

Vílchez (2005), expresa que el uso de las TIC implica el diseño de ambientes ricos en interactividad, en recursos multimediales en el que los docentes universitarios favorezcan el desarrollo de habilidades de pensamiento y en el que las nociones y conceptos de matemáticas sean más significativos

Las TIC en el proceso de enseñanza y aprendizaje de las matemáticas se pueden aplicar como recursos de apoyo, a través de diferentes software educativo, así como también desde el diseño de materiales digitales que incluyen, los materiales didácticos multimedia (Begoña, 2008) y los objetos de aprendizaje OA hallados en las bibliotecas digitales o repositorios, como en www.merlot.org, por ejemplo: Hippocampus Calculo I, que contiene videos explicativos con los temas de: trigonometría, calculo I, II, III, álgebra lineal, ecuaciones diferenciales, series infinitas.

Los materiales digitales para la enseñanza y el aprendizaje de las matemáticas y de las ciencias, se deben evaluar con el fin de conocer si se cumple con los estándares de calidad. De acuerdo a Cabero y Duarte (1999), en el material multimedia de se deben evaluar: el diseño técnico y las características tecnológicas incluidas la interactividad y uso del estudiante, el diseño desde el punto de vista didáctico, los contenidos, aspectos económicos, el contexto educativo. 
El Grupo de Investigación en Tecnología Educativa (GITE) de la Universidad de Murcia, propone una herramienta de evaluación pedagógica de material multimedia educativo con base en cinco dimensiones: identificación y análisis descriptivo, análisis didáctico, análisis psicopedagógico, aspectos económicos y valoración global (Prendes, 2003).

En este mismo sentido Barbera et al., (2008) señalan que los materiales digitales deben ser evaluados por usuarios referidos a los alumnos y docentes, expertos desde el punto de vista tecnológico y pedagógico. Narváez et al., (2008) proponen también la evaluación en la interacción productos, experto y usuarios.

El componente pedagógico es importante en el diseño y evaluación de los recursos digitales (Narváez et al., 2008; Sánchez, 2012), pero una limitación que presentan los materiales digitales elaborados para apoyar la enseñanza y aprendizaje de las ciencias, es la falta de un enfoque pedagógico que pueda generar materiales didácticos que inviten a resolver problemas con diferentes niveles de dificultades y la integración entre disciplinas (Franzolin et al., 2006); Como lo señala Guzmán (1993), se debe hacer énfasis en la comprensión de los procesos matemáticos, es decir, hacer un análisis y comprensión de los contenidos y no encerrarse en lo rutinario que desgasta y desorienta al alumno y más bien prepararlo para el dialogo inteligente con las herramientas existentes.

El objetivo de este trabajo fue el diseño de un material didáctico multimedia de matemáticas MDMM, basado en la integración del modelo pedagógico del aprendizaje significativo y la tecnología, con evaluación cualitativa por usuarios, un experto en contenidos y la evaluación cuantitativa del rendimiento académico de los estudiantes mediante la prueba t de Student de comparación de dos muestras independientes, a través de un examen de conocimientos entre un grupo de control y otro, que trabajó con el MDMM producido.

\section{MATERIALES Y MÉTODOS}

\section{Ubicación y muestra}

El estudio se realizó en la Universidad de los Llanos sede Barcelona, ubicada 12 kilómetros vía Puerto López, durante el primero y segundo periodo académico de 2011 con dos grupos de estudiantes del curso de Matemáticas I del Programa de Ingeniería Electrónica de la Facultad de Ciencias Básicas e Ingeniería. El grupo de control fueron 25 estudiantes, mientras que el grupo de prueba fue de 24 alumnos. Las dos muestras fueron jóvenes de estratos 1, 2 y 3 de la ciudad de Villavicencio y de otros municipios del departamento del Meta, con edades entre 16 a 20 años.

\section{Tipo de investigación}

La investigación fue de tipo cualitativo y experimental. En la parte cualitativa de acuerdo a Barbera et al., (2008) y a Narváez et al., (2008) el material multimedia producido se evaluó por parte del grupo de estudiantes de prueba y por un experto en contenidos. La evaluación cuantitativa se realizó mediante un examen de conocimientos por inferencia estadística a través de la prueba t de Student de comparación entre un grupo de control que no trabajó con el multimedia diseñado y un grupo de ensayo que practicó con el material digital desarrollado.

\section{Metodología para el desarrollo del material digital}

En el desarrollo del material didáctico multimedia se aplicó la metodología de ingeniería del software educativo propuesta por Galvis, (1992) que comprende las siguientes etapas: análisis, diseño, desarrollo, implementación por prueba piloto, prueba de campo y evaluación.

En la primera etapa se realizó un análisis de las dificultades que tienen los estudiantes para el aprendizaje de las matemáticas y una revisión en 
la literatura de las características y evaluación de software multimedia educativo desarrollado en matemáticas y en ciencias básicas.

En el diseño y desarrollo del material digital se tuvieron en cuenta los criterios y características como la interactividad y calidad técnica, el diseño gráfico, la navegabilidad, la flexibilidad, y la dinámicidad; propuestos por Cabero et al.,(2004) y Gisbert et al., (2004) y las actividades de aprendizaje y de evaluación del componente pedagógico y didáctico basadas en el aprendizaje significativo propuesto por Ausubel, que surge de la interacción de la información nueva con la estructura cognitiva de la persona que aprende (Rodríguez et al., 2008) correlacionando el componente tecnológico mediante la interactividad reflejada en la interacción docente-contenidos-alumno, que requieren este tipo de recursos (Mauri et al., 2005).

En esta parte se incluyó el diseño de la interfaz de inicio y la interfaz general, se digitaron en forma sintetizada los contenidos de los módulos, se integraron los objetivos de aprendizaje y las actividades de aprendizaje y de evaluación de cada módulo con imágenes, gráficas o tablas, información científica a través de vínculos, sonido, cuadros con texto, animaciones, vínculo a páginas web y las situaciones problemáticas, teniendo en cuenta el análisis de la primera etapa para favorecer el aprendizaje de los temas principales de matemáticas I. Las herramientas de software que se utilizaron para el desarrollo son las incluidas en la Creative Suite 5 de Adobe.

La implementación del material multimedia desarrollado se realizó mediante una prueba piloto con la aplicación del software educativo en las clases presenciales a una muestra de 24 estudiantes, del Programa de Ingeniería Electrónica que cursaban matemáticas I, durante el segundo periodo de 2011. A todos los estudiantes del curso de matemáticas I se les entregó una copia del material didáctico multimedia terminado.

\section{Evaluación cualitativa}

En la prueba de campo que se realizó con los estudiantes considerados como grupo experimental, se aplicó un instrumento de evaluación que indagó por las características principales sobre la presentación de la información, aspectos de la tecnología y aspectos pedagógicos, según valoración en la escala de Likert, así: totalmente de acuerdo = 4 ; de acuerdo $=3$; en desacuerdo $=2$; y totalmente en desacuerdo $=1$, (Pérez, 1994)

La evaluación por parte del experto en contenidos de matemáticas evaluó la calidad, idoneidad, cientificidad, actualización, coherencia y correspondencia de los contenidos, a través de un formato que incluyó la calidad, idoneidad, carácter científico, actualización, pertinencia y coherencia de los contenidos del cálculo diferencial.

\section{Evaluación cuantitativa}

El estudio cuantitativo se desarrolló por estadística inferencial con ayuda del programa Statistical Package for the Social Sciences SPSS, mediante la prueba t de Student de muestras independientes, a través de un examen de conocimientos sobre un total de 20 puntos, para conocer las diferencias en el rendimiento académico de los grupos de comparación: grupo de control o de enseñanza tradicional y el grupo experimental o que trabajó con el multimedia de matemáticas.

El cumplimiento de las condiciones de esta prueba paramétrica se verificó mediante las pruebas de normalidad de Shapiro-Wilk y la prueba de Levene de igualdad de varianzas, con la aplicación del paquete estadístico SPSS versión 19.0 en la opción de estadística descriptiva para la prueba de normalidad, la prueba t de muestras independientes en la comparación de medias para la prueba de homogeneidad de varianzas con la formulación de las respectivas hipótesis nula y alternativa, y el generador de gráficos en la representación de las medias de cada grupo (Castañeda et al., 2010). 
El estudio que combina el análisis de datos cualitativos y cuantitativos permite un enfoque más integral, completo y la profundización en el problema de investigación planteado, (Hernández et al., 2010).

\section{RESULTADOS Y DISCUSIÓN}

Se produjo un material didáctico multimedia de matemáticas, cuyos contenidos específicos del cálculo diferencial corresponden a los temas de las funciones, los límites, las derivadas y sus aplicaciones, con muchos ejemplos resueltos basados en el principio pedagógico del aprendizaje significativo, que de acuerdo a Moreira (2000) se fundamenta en llegar a conseguir la interacción entre el nuevo conocimiento y el conocimiento previo del que aprende, teniendo en cuenta el no uso del tablero, provocar un alumno activo, la aplicación de varias estrategias de enseñanza, la no centralización en el libro de texto y la diversidad de materiales educativos, principios que se relacionaron en el diseño del material digital producido a través de las diferentes animaciones y simulaciones centradas en el alumno, las cuales puede repetir o revisar cuantas veces necesite, según sus conocimientos de base, su capacidad de aprendizaje y sirve para promover su autonomía y autoaprendizaje.

En la imagen 1 se presenta la interfaz del material digital de matemáticas para un ejemplo de aplicación de la derivada, que se refiere a la producción de automóviles por año, a través del cual se pretende que el alumno mediante la repetición de la animación según su ritmo de aprendizaje comprenda y recuerde que la derivada en este caso es, la velocidad con que cambia la producción de automóviles con respecto al tiempo.

En la imagen 2 se muestra la simulación del material educativo desarrollado que incluye la razón de cambio, otro ejemplo de aplicación de la derivada que constituye un tema teórico complejo de comprensión para los alumnos, pero que con ayuda de la tecnología le facilita recoger los conocimientos previos, en este caso de la fórmula del volumen y del concepto de la derivada, que puede servir para determinar la razón de cambio o velocidad del radio respecto al tiempo, una situación cercana al mundo real en el que el estudiante encuentra sentido a las matemáticas y no simples ejercicios cerrados de lápiz y papel, propios de la enseñanza tradicional y que no consideran la estructura cognitiva del que aprende, (Ausubel, 1983).

Estudios publicados relacionados con software educativo o material digital que involucre la

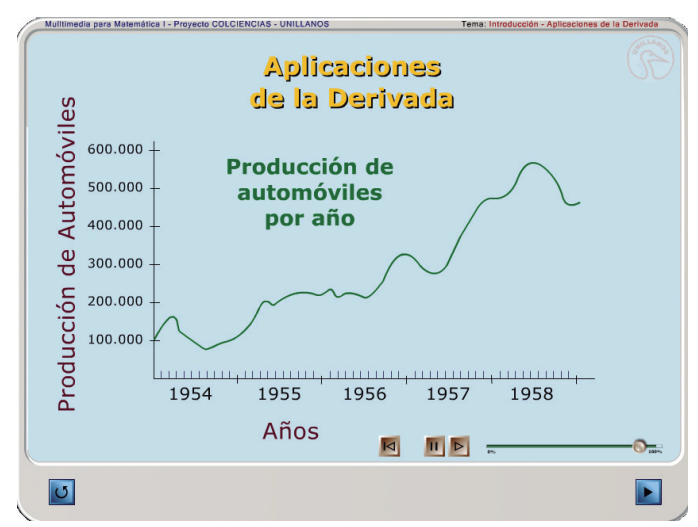

Imagen 1. Animación de una aplicación de la derivada

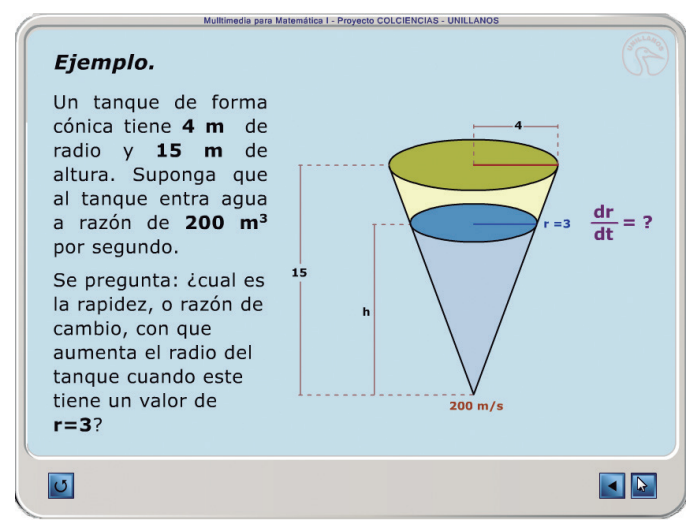

Imagen 2. Simulación de la razón de cambio 
integración de las matemáticas con el aprendizaje significativo y las TIC, no existen reportados, lo que se encuentran son resultados de investigaciones que aplican la tecnología en el diseño e implementación de este tipo de materiales en temas como la geometría, el álgebra, la derivada, la integral y el cálculo numérico (Camacho y Depool, 2003; Depool, 2004; Ferrara et al, 2006; Aranda y callejo, 2010).

El enfoque pedagógico del material desarrollado marca la diferencia respecto al software de matemáticas o en general del software educativo reportado en la literatura, que según evaluaciones no presentan definido este eje central (Cataldi, 2000; Vidal y González, 2002; Franzolin et al., 2006), que es el que debe dirigir todo material que tenga el objetivo de apoyar el proceso de enseñanza y aprendizaje.

El material didáctico multimedia de matemáticas generado contribuye de esta manera a que esta ciencia exacta sea vista con más significado, comprendida más fácilmente por el que aprende y sea una ayuda dinámica al problema del aprendizaje resaltado por varios autores.

\section{Evaluación cualitativa}

Con la prueba piloto que se realizó al material digital que se produjo, se logró a través de los formatos aplicados encontrar y corregir detalles y errores especialmente en la sincronización de algunas animaciones, simulaciones y mejorar el producto final.

Los resultados de la prueba de campo, en la que los usuarios calificaron el material desarrollado con un $90 \%$ de satisfacción respecto a que estuvieron de acuerdo y completamente de acuerdo según la escala de Likert, expresando que los contenidos facilitaban el aprendizaje de los diferentes conceptos de matemáticas como los limites, las derivadas; algunos estudiantes resaltaron que las gráficas de las funciones realizadas a través de las animaciones y las simulaciones permiten comprender a medida que se van construyendo las relaciones que existen entre la ecuación teórica y la curva dibujada.

Las observaciones de los alumnos consignadas al final del instrumento de evaluación, permiten relacionar la ventaja que ofrece la tecnología sobre el libro de texto, la diferencia del uso del pizarrón y acercarse a la línea del aprendizaje significado como lo destaca Rodríguez et al., (2008).

El experto en contenidos de matemáticas evaluó satisfactoriamente el material digital con la expresión de las siguientes afirmaciones de acuerdo al instrumento de evaluación diligenciado: en este material educativo se incluyen los temas del cálculo diferencial que normalmente se deben trabajar en un periodo académico a nivel universitario; los objetivos propuestos se correlacionan con los contenidos de cada tema; la información se presenta en forma coherente, científica y actualizada con lo que se puede generar una actitud positiva hacia la ciencia; señaló también que el diseño gráfico de las funciones ofrece la posibilidad de que el alumno tenga a su alcance fácilmente toda la información necesaria para asimilar este tema. El matemático sugiere una mayor cantidad de simulaciones en los diferentes temas del cálculo, para apoyar más al alumno, pero la experiencia en el diseño y desarrollo de un material digital implica unos recursos grandes que limitan especialmente la inclusión de las simulaciones, las cuales son muy costosas.

\section{Evaluación cuantitativa}

La aplicación de la prueba paramétrica t de Student requiere de que se compruebe el supuesto de normalidad, en el que la variable dependiente en los dos grupos presenta una distribución normal y saber si las diferencias de las medias de los dos grupos a comparar, grupo de control y grupo experimental, son significativas al $95 \%$ de probabilidad a través de la prueba de Levene en el que se asume igualdad de varianzas, (Castañeda et al., 2010; Pérez et al., 2012). 
En el paquete estadístico SPSS se seleccionó por estadística descriptiva la prueba de Shapiro-Wilk de muestras pequeñas iguales o inferiores a 50 sujetos, para conocer si la variable dependiente respecto a los dos grupos de comparación, el de enseñanza tradicional y el que trabajó con el multimedia, se ajustaban a una distribución normal, partiendo de la formulación de las siguientes hipótesis estadísticas con un nivel de significancia a de 0.05:

$\mathrm{H}_{0}$ : Los datos de las calificaciones del grupo tradicional se ajustan a una distribución normal.

$\mathrm{H}_{1}$ : Los datos de las calificaciones del grupo tradicional no se ajustan a una distribución normal.

$\mathrm{H}_{0}$ : Los datos de las calificaciones del grupo multimedia se ajustan a una distribución normal.

$\mathrm{H}_{1}$ : Los datos de las calificaciones del grupo multimedia no se ajustan a una distribución normal.

La conclusión de la prueba de normalidad de Shapiro-Wilk se puede observar en la figura 1, en la que el grado de significación p o sig del grupo del método de enseñanza tradicional, que aparece en la columna de color azul, presentó un valor de 0.577 mayor que el nivel de significancia $\alpha=0.05$; es decir, $p=0.577>0.05[D(25)=0.967, p>0.05]$, por lo que se acepta la hipótesis nula que se formuló: los datos de las calificaciones del grupo tradicional se ajustan a una distribución normal. En el caso del grupo que trabajó con el multimedia desarrollado, columna de color rojo, la significancia presentó un valor de 0.198 y por la misma regla de decisión, ahora [D (24)=0.944, p>0.05], se acepta la hipótesis nula: los datos de las calificaciones del grupo multimedia se ajustan a una distribución normal, (Pérez et al., 2012).

Confirmada la distribución normal de los dos grupos de estudio, se procedió a realizar la prueba t de Student para saber si existían diferencias en las medias de las calificaciones entre el grupo de enseñanza tradicional y el grupo que trabajó con el material multimedia producido, con la formulación

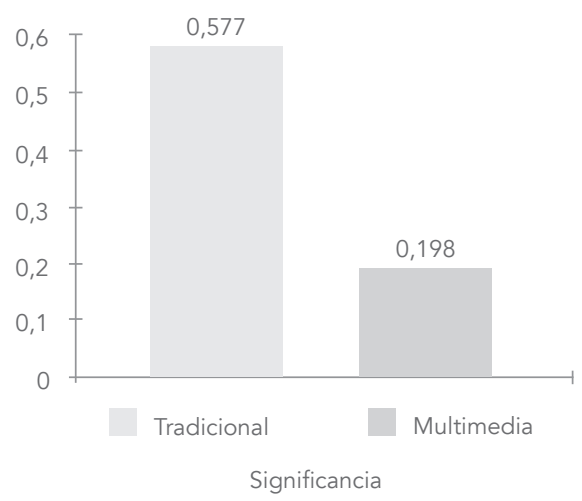

Figura 1. Representación de la conclusión de la prueba de normalidad de Shapiro-Wilk.

de las siguientes hipótesis estadísticas a un nivel de significancia $\alpha=0.05$ :

$\mathrm{H}_{0}$ : El nivel de conocimientos del cálculo diferencial es igual entre el grupo del método de enseñanza tradicional y el grupo que trabajó con el material multimedia desarrollado.

$\mathrm{H}_{1}$ : El nivel de conocimientos del cálculo diferencial es diferente entre el grupo del método de enseñanza tradicional y el grupo que trabajó con el material multimedia desarrollado.

En la tabla 1 se muestran los resultados de la media, desviación típica y error típico de la media de las calificaciones del grupo tradicional de enseñanza y el grupo multimedia. Se puede observar que el grupo experimental que trabajó con el multimedia obtuvo una media mayor con un valor de 13.83, mientras el grupo de enseñanza tradicional o de control alcanzó un valor de 9.44, pero surge la pregunta ¿La diferencia entre estas dos medias es estadísticamente significativa a un nivel a de 0.05 ?

Para responder a la pregunta anterior, se debe realizar primero la prueba de Levene con el fin de saber si se presentó o no igualdad de varianzas de los grupos de comparación, mediante la formulación de las siguientes hipótesis: 
Tabla 1. Estadísticos de grupo

\begin{tabular}{cccccc}
\hline & Método de Enseñanza & N & Media & Desviación típ. Error típ. de la media \\
\hline Calificación & TRADICIONAL & 25 & 9,44 & 3,720 &, 744 \\
\hline Examen & MULTIMEDIA & 24 & 13,83 & 3,632 &, 741 \\
\hline Conocimientos & & & & & \\
\hline
\end{tabular}

$H_{0}$ : Las varianzas entre el grupo de enseñanza tradicional y el grupo multimedia son iguales.

$H_{1}$ : Las varianzas entre el grupo de enseñanza tradicional y el grupo multimedia no son iguales

En la tabla 2 se presentan los resultados de la prueba de Levene, los cuales indican un grado de significación mayor que el nivel $\alpha$ es decir, $P=0.801>\alpha=$ 0.05 , por lo que se acepta la hipótesis nula de que las varianzas de los dos grupos son iguales y se observan los resultados de la prueba t de Student en la línea horizontal: se han asumido varianzas iguales, para su análisis correspondiente. La regla de decisión es, según (Castañeda et al., 2010): si el valor de P es menor que 0.05 , se considera significativo desde el punto de vista estadístico y como en este caso $\mathrm{P}=0.000<0.05$, se deduce que se presentaron diferencias estadísticamente significativas entre las medias de los dos grupos comparados y permite rechazar la hipótesis nula y se acepta la hipótesis alterna, es decir: El nivel de conocimientos de cálculo diferencial del grupo que trabajó con el material multimedia desarrollado es estadísticamente significativo con respecto al grupo del método de enseñanza tradicional.

El valor de $t$ de Student ( $t$ ), los grados de libertad ( $\mathrm{gl})$, el valor de significancia (P) y la diferencia de medias (DM) se presentan resumidas en la siguiente expresión, $[t=-4.181 ; g l=47 ; \mathrm{P}<0.01$ : $\mathrm{DM}=-4.393]$ de la tabla 3.

Tabla 2. Resultados de la prueba de Levene y t de Student

\begin{tabular}{|c|c|c|c|c|c|c|c|c|c|c|}
\hline & & $\begin{array}{r}\text { Pruel } \\
\text { Leven } \\
\text { la igua } \\
\text { varia }\end{array}$ & $\begin{array}{l}\text { oa de } \\
\text { e para } \\
\text { ldad de } \\
\text { anzas }\end{array}$ & & \multicolumn{6}{|c|}{ Prueba T para la igualdad de medias } \\
\hline & & \multirow[t]{2}{*}{$\mathrm{F}$} & \multirow[t]{2}{*}{ Sig. } & \multirow[t]{2}{*}{$\mathrm{t}$} & \multirow[t]{2}{*}{$g l$} & \multirow[t]{2}{*}{$\begin{array}{c}\text { Sig. } \\
\text { (bilateral) }\end{array}$} & \multirow[t]{2}{*}{$\begin{array}{l}\text { Diferencia } \\
\text { de medias }\end{array}$} & \multirow{2}{*}{$\begin{array}{l}\text { Error típ. } \\
\text { de la } \\
\text { diferencia }\end{array}$} & \multicolumn{2}{|c|}{$\begin{array}{c}95 \% \text { Intervalo de } \\
\text { confianza para la } \\
\text { diferencia }\end{array}$} \\
\hline & & & & & & & & & Inferior & Superior \\
\hline \multirow{2}{*}{$\begin{array}{c}\text { Calificación } \\
\text { de Examen } \\
\text { de Conoci- } \\
\text { mientos }\end{array}$} & $\begin{array}{l}\text { Se han } \\
\text { asumido } \\
\text { varianzas } \\
\text { iguales }\end{array}$ & 0,064 & 0,801 & $-4,181$ & 47 & 0,000 & $-4,393$ & 1,051 & $-6,507$ & $-2,279$ \\
\hline & $\begin{array}{l}\text { No se han } \\
\text { asumido } \\
\text { varianzas } \\
\text { iguales }\end{array}$ & & & $-4,183$ & 46,985 & 0,000 & $-4,393$ & 1,050 & $-6,506$ & $-2,280$ \\
\hline
\end{tabular}


En la figura 2 se ilustran los valores promedio de las calificaciones del examen de conocimientos sobre 20 puntos obtenidos por los dos grupos de comparación, realizada con el programa estadístico SPSS, en la que se puede observar que el grupo experimental que trabajó con el material multimedia diseñado obtuvo una media de 13.83 aproximada a 14 y el grupo de control o de enseñanza tradicional alcanzó una media de 9.44 aproximada a 9.

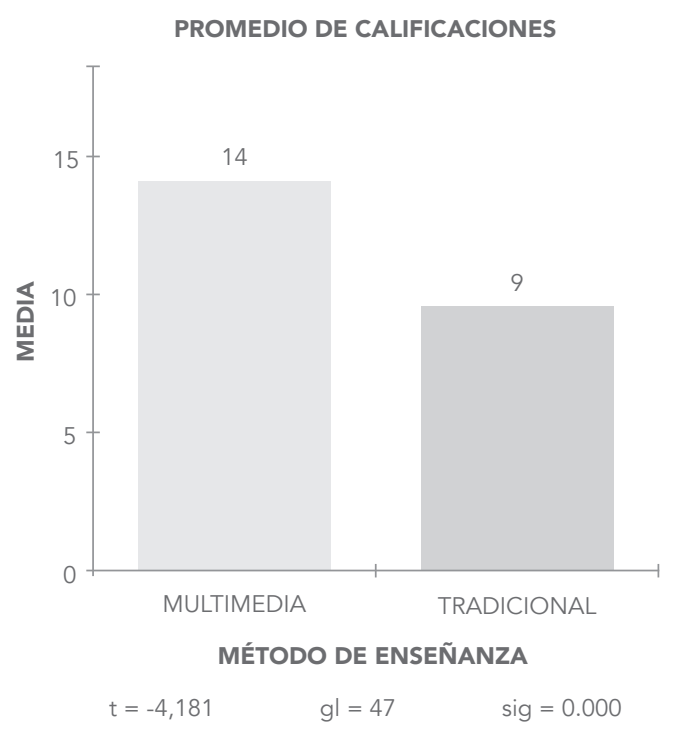

Figura 2. Representación de los promedios de los dos grupos comparados.

\section{CONCLUSIÓN}

La correlación de los resultados de la evaluación cualitativa realizados por los alumnos y el experto en contenidos, con los resultados de la evaluación cuantitativa, permite resaltar un favorecimiento hacia el aprendizaje significativo del cálculo diferencial, en el que una estrategia de enseñanza apoyada en la tecnología, diferente a la tradicional que se basa en el libro de texto y el pizarrón, muy de acuerdo a lo señalado por Moreira, (2000) y Rodríguez et al., (2008), ofrece un apoyo importante al proceso de enseñanza y aprendizaje de una ciencia exacta tan compleja como es la matemática.

En este sentido un material didáctico multimedia o un software educativo u objeto de aprendizaje, no solo debe cumplir con unas características técnicas determinadas, sino que debe tener un enfoque pedagógico bien definido, orientado por unos objetivos, actividades de enseñanza aprendizaje y actividades de evaluación interactivas, que dirija el material digital hacia un fin educativo productivo para hacer la diferencia de muchos software evaluados sin este componente como lo muestran algunos autores (Vidal y González, (2002); Franzolin et al., (2006); Narváez et al., (2008).

Se destaca el apoyo que ofrecen las simulaciones tanto en el diseño de materiales digitales, como en la parte de la evaluación que permite la interacción con el usuario, con múltiples opciones para la investigación y exploración de la aplicación de otros modelos pedagógicos, pero tienen la limitante de que son muy costosas, haciendo que este tipo de recursos en su conjunto impliquen un trabajo interdisciplinario con un alto costo en recurso humano y económico.

\section{AGRADECIMIENTOS}

Agradecemos el apoyo financiero de Colciencias y de la Universidad de los Llanos por la contrapartida en otros recursos.

ISSN 1900-4699 • Volumen 11 • Número 1 • Páginas 70-83 • 2015 


\section{REFERENCIAS}

1. Aranda C y Callejo, M.L. 2010. Construcción del concepto de dependencia lineal en un contexto de geometría dinámica: Un estudio de casos. Revista Latinoamericana de Investigación en Matemática Educativa, 13(2):129-158.

2. Ausubel D, Novak J y Hanesian, H. 1983. Psicología Educativa: Un punto de vista cognoscitivo. Editorial Trillas, México, 623 p.

3. Barberá E, Mauri T y Onrubia J et al. 2008. Cómo valorar la calidad de la enseñanza basada en las TIC. Pautas e instrumentos de análisis. Ed. Graó, Barcelona, 83 p.

4. Berry, J. S., y Nyman, M. A. 2003. Promoting Students' graphical understanding of the calculus. Journal of Mathematical Behavior, 22:481-497.

5. Cabero J y Duarte A. 1999. Evaluación de medios y materiales de enseñanza en soporte multimedia. Pixel-Bit. Revista de Medios y Educación, 13:23-45.

6. Cabero, J.; Morales, J. A.; Barroso, J.; Román, P.; Romero, R. 2004. La red como instrumento de formación. Bases para el diseño de materiales didácticos. Pixelbit. Revista de Medios y Educación, 22:5-23.
7. Camacho, M. y Depool, R. 2002. Students' attitudes towards Mathematics and computers when using DERIVE in the learning of calculus concepts. International Journal of Computer Algebra in Mathematics Education, 9(4):259-283.

8. Camacho, M. y Depool, R. 2003. Using DERIVE to understand the concept of Definite Integral [107 párrafos]. International Journal for Mathematics Teaching and Learning, 10:1-16

9. Camacho, M. 2005. La enseñanza y aprendizaje del análisis matemático hacienda uso de CAS (computer algebra system). En A. Maz, B. Gómez y M. Torralbo, (Eds.), Investigación en Educación Matemática IX : 97-110. Córdoba: SEIEM.

10. Castañeda M.B, Cabrera A, Navarro Y y Vries W. 2010. Procesamiento de datos y análisis estadístico utilizando SPSS. Edipucrs, Porto Alegre: 55-62 p

11. Cataldi, Z. 2000. Metodología de diseño, desarrollo y evaluación de software educativo. Tesis de Maestría, Facultad de Informática, Universidad Nacional de la Plata, Argentina.

12. Depool, R. 2004. La enseñanza y aprendizaje del Cálculo Integral en un entorno 
computacional. Actitudes de los estudiantes hacia el uso de un Programa de Cálculo Simbólico (PCS). Servicio de Publicaciones de la Universidad de La Laguna. Tesis Doctoral. España.

13. Ferrara F, Pratt D y Robutti O. 2006. The role and uses of technologies for the teaching of algebra and calculus. En A. Gutierrez y P. Boero (Eds.), Handbook of Research on the Psychology of Mathematics Education. Past, Present and Future pp. 237-274. Rotterdam/ Taipei: Sense Publishers.

14. Galvis, A. 1992. Ingeniería del Software Educativo. Ediciones Universidad de los Andes. Bogotá. 64.82 p.

15. Gisbert, M, Salinas, J, Chan, M.E y Guardia L. 2004. Conceptualización de materiales multimedia. Ed. Eureka media, España, 34 p.

16. Franzolin F, Pereira A P, Pereira I y Fejes M. 2006. Algunas consideraciones sobre los aspectos pedagógicos de software para la enseñanza de las ciencias. Revista de educación en ciencias 7 (1): 1-16.

17. Hernández R, Fernández $C$ y Baptista M P. 2010. Metodología de la Investigación. Quinta edición. McGrawHill, México, 544-578 p.
18. Kendal, M. 2001. Teaching and learning introductory differential calculus with a computer algebra system. Submitted in total fulfilment of the requirements of the degree of Doctor of Philosophy. Department of Science and Mathematics Education the University of Melbourne.

19. Kirk S.A y Bateman, B. 1962. Diagnosis and remediation of learning desabilities. Exceptional Children, 29: 73-78.

20. Kumar, T. 2007. Computer graphics as an instructional aid in an introductory differential calculus course. International Electronic Journal of Mathematics Education, (2)1: 32-48.

21. Kutzler, B. 2003. CAS as pedagogical tools for teaching and learning mathematics. En Fey et al. (eds.), Computer algebra systems in secondary school education (pp. 53-71).

22. Jordan C. N y Hanich B. 2000. Mathematical thinking in second grade children with different forms of LD. Journal of Learning Disabilities, 33: 567-578.

23. Landerl K, Bevan A y Butterworth B. 2004. Developmental dyscalculia and basic numerical capacities: A study of 8-9-year-old Students. Cognition, 93: 99-125. 
24. Mazzoco M. M. y Myers, G. F. 2003. Complexities in identifying and defining mathematics learning disability in the primary school-age years. Annals of Dyslexia, 53: 218-253.

25. Moreira M A. 2000. Aprendizaje significativo: teoría y práctica. Visor, Madrid, 100 p.

26. Narvaez C, Martinez M, Vargas $P$ y Goset J. 2008. Desarrollo y evaluación de un CD multimedia de aplicación para la resolución de casos en ciencias básicas. Journal of Science Education 9(2): 51-54.

27. Pérez, G. 2004. Investigación cualitativa. Retos e interrogantes. 2 Vol. Ed. La Muralla Madrid, $72 \mathrm{p}$.

28. Pérez R, García J.L, Gil J.A y Galán A. 2012. Métodos y diseños de investigación en Educación. UNED - Pearson, Madrid, 289-331 p.

29. Rodríguez M L, Moreira M A, Caballero M C y Greca I. 2008. La teoría del aprendizaje significativo en la perspectiva de la psicología cognitiva. Editorial Octaedro. Barcelona. 7-44 p.

30. Sánchez, M. M. 2012. Diseño de recursos digitales para entornos de e-learning en la enseñanza universitaria. RIED. Revista Iberoamericana de Educación a Distancia, 15, (2): 53-74.
31. Vidal, G. y González, H. 2002. Evaluación pedagógica del simulador del laboratorio químico modelchemlab. Revista pedagogía universitaria, 7 (4): 17-29

32. Vílchez E. 2005. Impacto de las Nuevas Tecnologías de la Información y la Comunicación para la enseñanza de la Matemática en la Educación Superior. Universidad Nacional Escuela de Matemática Centro de Investigación y Docencia en Educación. Revista Digital Matemática, Educación e Internet (www. cidse.itcr.ac.cr), Volumen 7, número 2.

33. Villarreal, M. 2003. Pensamiento matemático, cálculo diferencial y computadoras. Educación Matemática, 15(1): 99-122.

\section{CONSULTA PAGINAS WEB}

1. Begoña B. 2008. Elaboración y desarrollo de un libro multimedia sobre programación matemática. Revista de innovación educativa. 1: 56-62 Disponible en: http://ojs.uv.es/index. php/attic/article/view/36/36. Consultado el 18 de julio de 2014.

2. Guzmán O. M. y Gil Pérez D. 1993. Enseñanza de las ciencias y de la matemática. 
Tendencias e Innovaciones. Organización de Estados Iberoamericanos para la Educación, la Ciencia y la Cultura. Editorial Popular. ISBN: 84-7884-092-3. Disponible en http://www.oei. org.co/oeivirt/ciencias.htm\#Indice. Consultado el 12 de agosto de 2014.

3. Martínez F, Prendes M, Alfageme M, Amorós L, Rodríguez $T$ y Solano I. Herramienta de evaluación de multimedia didáctico. Disponible en: http://ocw.um.es/gat/contenidos/mpaz/tema4/evaluacionMMdidactico. pdf. Consultado el 12 de junio de 2014.

4. Mauri T, Onrubia J, Coll Cy Colomina R. 2005. La calidad de los contenidos educativos reutilizables: diseño, usabilidad y prácticas de uso. Revista de educación a distancia, año IV. Disponible en: http://www.um.es/ead/red/M2/. Consultado el 12 de julio de 2014.

5. Ministerio de Educación Nacional. 2013. Disponible en: http://www.mineducacion.gov.co/ sistemasdeinformacion/1735/articles-254702_ ciencias_basicas_desercion.pdf. Consultado el 10 de julio de 2014.

6. http://www.colombiaaprende.edu.co/html/ micrositios/1752/w3-article-343426.html. Consultado el 14 de diciembre de 2014.
7. Moreira M. C. 2005. Aprendizaje significativo crítico. Indivisa. Boletin de estudios e investigación. 6. 82-102 p. http://www.redalyc.org/ pdf/771/77100606.pdf

8. Objeto de aprendizaje Hippocampus calculo I. www.merlot.org. Disponible en: http://www. hippocampus.org/HippoCampus/Calculus\%20\%26\%20Advanced\%20Math Consultado el 10 agosto de 2014.

9. Prendes, M. P. 2003. Diseño de cursos y materiales para telenseñanza. Simposium Iberoamericano de Virtualización del Aprendizaje y la Enseñanza. Costa Rica. [En línea] Disponible en: http:// tecnologiaedu.us.es/ bibliovir/pdf/paz5.pdf. Consultado el 15 de agosto de 2014 . 